TRANSACTIONS OF THE

AMERICAN MATHEMATICAL SOCIETY

Volume 354, Number 10, Pages 4043-4065

S 0002-9947(02)03023-4

Article electronically published on May 22, 2002

\title{
SPECTRAL ASYMPTOTICS FOR STURM-LIOUVILLE EQUATIONS WITH INDEFINITE WEIGHT
}

PAUL A. BINDING, PATRICK J. BROWNE, AND BRUCE A. WATSON

Abstract. The Sturm-Liouville equation

$$
-\left(p y^{\prime}\right)^{\prime}+q y=\lambda r y \text { on }[0, l]
$$

is considered subject to the boundary conditions

$$
\begin{gathered}
y(0) \cos \alpha=\left(p y^{\prime}\right)(0) \sin \alpha, \\
y(l) \cos \beta=\left(p y^{\prime}\right)(l) \sin \beta .
\end{gathered}
$$

We assume that $p$ is positive and that $p r$ is piecewise continuous and changes sign at its discontinuities. We give asymptotic approximations up to $O(1 / \sqrt{n})$ for $\sqrt{\lambda_{n}}$, or equivalently up to $O(\sqrt{n})$ for $\lambda_{n}$, the eigenvalues of the above boundary value problem.

\section{INTRODUCTION}

Asymptotics for Sturm-Liouville equations

$$
-\left(p y^{\prime}\right)^{\prime}+q y=\lambda r y
$$

subject to boundary conditions

$$
\begin{aligned}
y(0) \cos \alpha & =\left(p y^{\prime}\right)(0) \sin \alpha, \quad \alpha \in(-\pi / 2, \pi / 2], \\
y(l) \cos \beta & =\left(p y^{\prime}\right)(l) \sin \beta, \quad \beta \in(-\pi / 2, \pi / 2],
\end{aligned}
$$

are classical when the coefficients permit a Liouville transformation (so, in particular, $p, r$ are positive). For example, if the resulting $q$ (once $p$ and $r$ are reduced to one) is $L^{1}$ and $\alpha \beta \neq 0$, then the eigenvalues (which we label $\lambda_{m}$, corresponding to eigenfunctions with $m$ zeros in $(0, l)$ for sufficiently large $m$ ) satisfy

$$
\lambda_{m}=a m^{2}+b+o(1)
$$

as $m \rightarrow \infty$, where $a$ and $b$ can be given simply in terms of the data in (1.1)-(1.3). We remark that (1.4) is sometimes written in the equivalent form

$$
s_{m}=c m+d m^{-1}+o\left(m^{-1}\right) .
$$

Here $s_{m}$ is (for large $m$ ) the positive square root of $\lambda_{m}$. When more smoothness is imposed on the coefficients, the $o$ terms may be developed in powers of $m^{-1}$. See, e.g., 8], [10] for discussions of these ideas.

Received by the editors January 12, 2002.

2000 Mathematics Subject Classification. Primary 34L20, 34B09, 34B24; Secondary 47E05.

Key words and phrases. Eigenvalue asymptotics, indefinite weight, turning point.

(C)2002 American Mathematical Society 
Weaker results are available when less is assumed of the coefficients. The most general result of this type seems to be due to Atkinson and Mingarelli [1], who assume that $1 / p, q, r \in L^{1}, p>0$, with no sign restriction on $r$. Then (for large $m$ ) the eigenvalues form two sequences $\lambda_{m}^{ \pm}$, one positive and one negative, tending to $\pm \infty$, and the positive one satisfies an asymptotic relation of the form

$$
\lambda_{m}^{+}=a^{+} m^{2}+o\left(m^{2}\right) .
$$

It is important to note that $a^{+}$depends only on the positive values of $r$; so one can "ignore" negative values. We remark that the corresponding results for the sequence of negative eigenvalues can be obtained by considering only negative values of $r$, by virtue of the relation $\lambda r=(-\lambda)(-r)$. In view of this we shall consider only the positive sequence in what follows.

We shall examine a situation in between those of the previous two paragraphs, where $r$ is permitted sign changes at a finite number of so-called "turning points" (TP). When the coefficients are smooth (in particular at the TP which we then call smooth TP), asymptotics have been developed by R. E. Langer [9] and several authors since (see, e.g., [4] and the references there). By contrast, our coefficients (at least $p r$ ) will be discontinuous at the TP. Actually, Dorodnicyn [4] does allow a type of singularity at his (single) TP, but his results do not allow finite discontinuities as here. Recently Strelitz [11] has treated a situation where both smooth and discontinuous TP are permitted. While his results are mostly for more general problems depending on a large parameter $\lambda$, his asymptotics for eigenvalues reduce to (1.6) for the case studied here.

An improvement on (1.6) has been obtained by Eberhard and Freiling [5], for the case when $p=1$ and $r$ is a step function; so all TP are discontinuous. They allow more general differential equations and boundary conditions, but for (1.1)-(1.3), their asymptotic relation takes the form

$$
\lambda_{m}^{+}=a^{+} m^{2}+O(m)
$$

which is equivalent to

$$
s_{m}=c m+O(1)
$$

for the corresponding square roots. Here we shall allow all the coefficients to be nonconstant between the TP, and we shall obtain the $O(1)$ term in (1.8) explicitly, with an error term of $O\left(m^{-1 / 2}\right)$. A comparison of (1.5) with (1.8) shows that indefiniteness of the weight function $r$ gives rise to the extra terms between $\mathrm{cm}$ and $d m^{-1}$. In the sequel, for brevity, we also refer to $s_{m}=\sqrt{\lambda_{m}}$ as an eigenvalue of (1.1)-(1.3).

It turns out that our $O(1)$ term is also independent of the negative values of $r$; so, up to $O\left(\mathrm{~m}^{-1 / 2}\right)$, the asymptotics depend only on subproblems on the disjoint intervals where $r$ is positive. In fact, our main result states (roughly) that the $\lambda_{m}^{+}$ asymptotically form the "disjoint" (see below) union of the positive eigenvalues for these subproblems. Two remarks are in order here, however. First, some of the eigenvalues from the subproblems may coincide. This resonance effect complicates our analysis considerably. For example, we must allow repeated elements in the above union (which is termed disjoint) in this case; see Definition 1.1. In particular, there is the possibility that an infinite sequence of $\lambda_{m}^{+}$appear asymptotically as multiple eigenvalues, despite the well-known fact that they are simple. We give an example of this behaviour in Section 6, where various sequences of eigenvalues 
are separated by distances that are exponentially small in $m$. The implications for numerical calculation are evident.

Our second remark concerns the fact that the subproblems have their own boundary conditions, and these turn out to depend on $s(=\sqrt{\lambda})$, except for the end intervals, where the original conditions (1.2) and (1.3) are used. We note that applications of $s$-dependent boundary conditions can be found in [6] and the references therein.

Section 2 gives the definition of, and some preliminary results about, a modified Prüfer angle. We develop asymptotics for problems with $s$-dependent boundary conditions on a single subinterval in Section 3. Section 4 extends the theory of the modified Prüfer angle to several subintervals. The proof of our main result, Theorem 1.2 below, is detailed in Section 5 .

Here and in the remainder of this work we assume that $0=a_{0}<a_{1}<\ldots<$ $a_{n}=l$, where $\left.p r\right|_{\left(a_{j}, a_{j+1}\right)}$ and $\left.(p r)^{\prime}\right|_{\left(a_{j}, a_{j+1}\right)}$ have continuous extensions to $\left[a_{j}, a_{j+1}\right]$, $\left.(p r)^{\prime \prime}\right|_{\left(a_{j}, a_{j+1}\right)} \in L^{1}\left(a_{j}, a_{j+1}\right)$ for all $j=0, \ldots, n-1$,

$$
\lim _{x \rightarrow a_{j}^{+}} \operatorname{pr}(x) \lim _{x \rightarrow a_{j}^{-}} \operatorname{pr}(x)<0, \quad j=1, \ldots, n-1,
$$

and $\lim _{x \rightarrow a_{0}^{+}} \operatorname{pr}(x) \neq 0 \neq \lim _{x \rightarrow a_{n}^{-}} \operatorname{pr}(x)$. In addition we assume $q \in L^{1}[0, l]$. By means of a standard change of independent variable (cf. [2]) we may and shall assume that $p=1$ in what follows.

With reference to (1.1), we define positive quantities

$$
\begin{aligned}
\delta_{j} & =\left[\frac{r\left(a_{j}^{+}\right)}{r\left(a_{j+1}^{-}\right)}\right]^{1 / 4}, \\
\rho_{j} & =\left|\frac{r\left(a_{j+1}^{-}\right)}{r\left(a_{j+1}^{+}\right)}\right|^{1 / 2}, \\
\epsilon_{j} & =\left|r\left(a_{j}^{+}\right)\right|^{1 / 2} \\
\nu_{j} & =\left|r\left(a_{j+1}^{-}\right)\right|^{-1 / 2}, \\
\zeta_{j} & =\int_{a_{j}}^{a_{j+1}} \sqrt{|r(x)|} d x, \quad j=0, \ldots, n-1,
\end{aligned}
$$

where $a_{j}^{+}$and $a_{j+1}^{-}$denote limits from respectively the right at $a_{j}$ and from the left at $a_{j+1}$, and we set $a_{n}^{+}=a_{n}^{-}$. Note that $\nu_{j}=\delta_{j}^{2} / \epsilon_{j}$ and $\rho_{j}=\left(\epsilon_{j+1} \nu_{j}\right)^{-1}$.

Definition 1.1. Given strictly increasing real sequences $\sigma_{0}, \ldots, \sigma_{m}$, each of which has no finite accumulation point, we say that a sequence $\sigma$ is the disjoint union

$$
\bigcup_{j=0, \ldots, m}^{\bullet} \sigma_{j}
$$

to order $\gamma$ if, with the sets $\sigma$ and $\bigcup\left\{\sigma_{j}: j=0, \ldots, m\right\}$ labelled $s_{0}, s_{1}, \ldots$ and $S_{0}, S_{1}, \ldots$ respectively in increasing order (but with each $S_{j}$ being repeated according to the number of $\sigma_{j}$ to which it belongs), there exist constants $\kappa, \kappa_{1}, \kappa_{2}$ such that for all $k \geq \kappa$,

$$
\left|s_{k}-S_{k+\kappa_{2}}\right|<\kappa_{1} k^{\gamma}
$$


If $r<-c<0$ on $\left(a_{j}, a_{j+1}\right)$, we define $\sigma_{j}=\emptyset$. If $r>c>0$ on $\left(a_{j}, a_{j+1}\right)$, we define $\sigma_{j}$ to be the set of positive eigenvalues $s=\sqrt{\lambda}$ of (1.1) with boundary conditions (1.2) and (1.10) for $j=0$, (1.9) and (1.10) for $1 \leq j \leq n-2$, and finally (1.9) and (1.3) for $j=n-1$, where

$$
\begin{aligned}
s y\left(a_{j}\right) & =\nu_{j-1} y^{\prime}\left(a_{j}\right), \\
s \epsilon_{j+1} y\left(a_{j+1}\right) & =-y^{\prime}\left(a_{j+1}\right) .
\end{aligned}
$$

Our principal result expresses $\sigma$ asymptotically in terms of the $\sigma_{j}$ as follows.

Theorem 1.2. The set $\sigma$ of positive eigenvalues $s=\sqrt{\lambda}$ of (1.1), (1.2) and (1.3) is the disjoint union of the $\sigma_{j}, j=0, \ldots, n-1$, defined as above, to order $-1 / 2$.

Asymptotic expressions for the sequences $\sigma_{j}$ are given in Corollary 3.6

\section{Preliminary considerations}

We start with the following.

Lemma 2.1. (a) If $r>c>0$ on $\left(a_{j}, a_{j+1}\right)$, where $c$ is a constant, then

$$
\left[\begin{array}{c}
y\left(a_{j+1}\right) \\
\frac{y^{\prime}\left(a_{j+1}\right)}{s}
\end{array}\right]=\left(\left[\begin{array}{cc}
\delta_{j} \cos \zeta_{j} s & \frac{\delta_{j}}{\epsilon_{j}} \sin \zeta_{j} s \\
-\frac{\epsilon_{j}}{\delta_{j}} \sin \zeta_{j} s & \frac{1}{\delta_{j}} \cos \zeta_{j} s
\end{array}\right]+O\left(\frac{1}{s}\right)\right)\left[\begin{array}{c}
y\left(a_{j}\right) \\
\frac{y^{\prime}\left(a_{j}\right)}{s}
\end{array}\right] .
$$

(b) If $r<c<0$ on $\left(a_{j}, a_{j+1}\right)$, where $c$ is a constant, then

$$
\left[\begin{array}{c}
y\left(a_{j+1}\right) \\
\frac{y^{\prime}\left(a_{j+1}\right)}{s}
\end{array}\right]=\left(\left[\begin{array}{cc}
\delta_{j} & \frac{\delta_{j}}{\epsilon_{j}} \\
\frac{\epsilon_{j}}{\delta_{j}} & \frac{1}{\delta_{j}}
\end{array}\right]+O\left(\frac{1}{s}\right)\right)\left[\begin{array}{l}
y\left(a_{j}\right) \\
\frac{y^{\prime}\left(a_{j}\right)}{s}
\end{array}\right] \cosh \zeta_{j} s .
$$

Here and below, $o(\cdot)$ and $O(\cdot)$ refer to the limiting process $s \rightarrow \infty$.

Proof. This is a direct consequence of the asymptotics in the Appendix of 7 (which treats $r>0$ and $\lambda \in \mathbb{C}$ ): (a) and (b) follow by taking positive and negative $\lambda$ respectively.

The following function enables us to write some tedious arithmetic in a compact form.

Definition 2.2. We denote by $\Theta(\omega ; k), k>0$, the angle depending continuously on $\omega$ such that $\Theta(0 ; k)=0$ and $\tan \Theta(\omega ; k)=k \tan \omega$.

Lemma 2.3. (a) $\Theta\left(\frac{m \pi}{2} ; k\right)=\frac{m \pi}{2}$ for all $m \in \mathbb{Z}$.

(b) If $\omega \in\left[\frac{(m-1) \pi}{2}, \frac{m \pi}{2}\right]$, then $\Theta(\omega ; k) \in\left[\frac{(m-1) \pi}{2}, \frac{m \pi}{2}\right]$, for all $m \in \mathbb{Z}$.

(c) $\Theta\left(\Theta(\omega ; k) ; \frac{1}{k}\right)=\omega$.

(d) $\Theta(\omega ; k)$ is $C^{\infty}$ with respect to both $\omega$ and $k$.

(e) For each $k>0$ there exist constants $c_{1}, c_{2}>0$ such that $c_{1} \leq \frac{\partial \Theta(\omega ; k)}{\partial \omega} \leq c_{2}$ for all $\omega \in \mathbb{R}$, and hence $\Theta(\omega, k)$ is monotonically increasing with respect to $\omega$ for fixed $k>0$.

(f) $\Theta(m \pi+\omega ; k)=m \pi+\Theta(\omega ; k)$ for all $m \in \mathbb{Z}$.

Proof. (a)-(c) and (f) are immediate. Moreover, $\frac{\partial \Theta(\omega ; k)}{\partial \omega}=k^{-1} \cos ^{2} \Theta \sec ^{2} \omega=$ $k^{-1} \cos ^{2} \Theta+k \sin ^{2} \Theta$, from which (d) and (e) follow easily.

The next item we introduce is a modified Prüfer angle. For details on the customary Prüfer angle we refer the reader to [3], and for different modifications of the Prüfer angle, to [1], [2]. 
Definition 2.4. We define the modified Prüfer angle, $\Omega(x ; s ; \chi ; \omega)$ of (1.1), to be the angle continuously dependent on $x$ such that

$$
\Omega(\chi ; s ; \chi ; \omega)=\omega, \quad \tan (\Omega(x ; s ; \chi ; \omega))=\frac{s y(x)}{y^{\prime}(x)},
$$

where $y$ is the solution of (1.1) satisfying the Cauchy conditions $y(\chi)=\sin \omega$ and $y^{\prime}(\chi)=s \cos \omega$.

Lemma 2.5. The modified Prüfer angle $\Omega$ has the following properties in common with the usual Prüfer angle:

(a) $\Omega(x ; s ; \chi ; \omega+m \pi)=\Omega(x ; s ; \chi ; \omega)+m \pi$ for all $m \in \mathbb{Z}$;

(b) $\Omega(x ; s ; \chi ; \omega)$ is monotonically increasing in $\omega$.

Proof. $\Omega$ is the solution of the differential equation

$$
\frac{\partial \Omega}{\partial x}=s\left[\cos ^{2} \Omega+\left(r-\frac{q}{s^{2}}\right) \sin ^{2} \Omega\right]
$$

with Cauchy condition $\Omega(\chi ; s ; \chi ; \omega)=\omega$. Now (a) (resp. (b)) follows because the right side of (2.4) is periodic (resp. Lipschitz) in $\Omega$.

The theorem below gives some basic building-blocks of the paper. We assume that the modified Prüfer angle at $a_{j}$ is $\Omega_{j}$, so in particular, $\Omega_{j+1}=\Omega\left(a_{j+1} ; s ; a_{j} ; \Omega_{j}\right)$.

Theorem 2.6. (a) If $r>c>0$ on $\left(a_{j}, a_{j+1}\right)$, where $c$ is a constant, then

$$
\Omega_{j+1}=\Theta\left(\zeta_{j} s+\Theta\left(\Omega_{j} ; \epsilon_{j}\right) ; \nu_{j}\right)+O\left(\frac{1}{s}\right) \text {. }
$$

(b) Let $\delta>0$ and $r<c<0$ on $\left(a_{j}, a_{j+1}\right)$, where $c$ is a constant. If

$$
\Omega_{j} \notin I(j ; s ; \delta)=\bigcup_{m \in \mathbb{Z}} I_{m}(j ; s ; \delta),
$$

where

$$
I_{m}(j ; s ; \delta)=\left(m \pi+\Theta\left(-\frac{\pi}{4} ; \frac{1}{\epsilon_{j}}\right)-\frac{\delta}{\sqrt{s}}, m \pi+\Theta\left(-\frac{\pi}{4} ; \frac{1}{\epsilon_{j}}\right)+\frac{\delta}{\sqrt{s}}\right),
$$

then for suitable constants $L_{j}(\delta)>0$,

$$
\Omega_{j+1} \in J(j ; s ; \delta)=\bigcup_{m \in \mathbb{Z}} J_{m}(j ; s ; \delta),
$$

where

$$
J_{m}(j ; s ; \delta)=\left[m \pi+\Theta\left(\frac{\pi}{4} ; \nu_{j}\right)-\frac{L_{j}(\delta)}{\sqrt{s}}, m \pi+\Theta\left(\frac{\pi}{4} ; \nu_{j}\right)+\frac{L_{j}(\delta)}{\sqrt{s}}\right]
$$

for large $s$.

Proof. For the proof we use the results of Lemma 2.1 From the definition of $\Theta(\cdot ; \cdot)$ and since $\Omega_{j}$ is the modified Prüfer angle at $a_{j}$, we have $y\left(a_{j}\right)=\rho \sin \Theta\left(\Omega_{j} ; \epsilon_{j}\right)$ and $\frac{y^{\prime}\left(a_{j}\right)}{s}=\rho \epsilon_{j} \cos \Theta\left(\Omega_{j} ; \epsilon_{j}\right)$ for some non-zero $\rho$.

For (a) we substitute the above expressions for $y\left(a_{j}\right)$ and $\frac{y^{\prime}\left(a_{j}\right)}{s}$ into (2.1), giving

$$
\left[\begin{array}{c}
y\left(a_{j+1}\right) \\
\frac{y^{\prime}\left(a_{j+1}\right)}{s}
\end{array}\right]=\rho\left[\begin{array}{c}
\delta_{j} \sin \left(\zeta_{j} s+\Theta\left(\Omega_{j} ; \epsilon_{j}\right)\right)+O(1 / s) \\
\frac{\epsilon_{j}}{\delta_{j}} \cos \left(\zeta_{j} s+\Theta\left(\Omega_{j} ; \epsilon_{j}\right)\right)+O(1 / s)
\end{array}\right],
$$

and hence (2.3) follows. 
Case (b) requires more careful analysis. From (2.2) we obtain

$$
\left[\begin{array}{c}
y\left(a_{j+1}\right) \\
\frac{y^{\prime}\left(a_{j+1}\right)}{s}
\end{array}\right]=\left[\begin{array}{c}
\delta_{j}\left(\sin \Theta\left(\Omega_{j} ; \epsilon_{j}\right)+\cos \Theta\left(\Omega_{j} ; \epsilon_{j}\right)+O(1 / s)\right) \\
\frac{\epsilon_{j}}{\delta_{j}}\left(\sin \Theta\left(\Omega_{j} ; \epsilon_{j}\right)+\cos \Theta\left(\Omega_{j} ; \epsilon_{j}\right)+O(1 / s)\right)
\end{array}\right] \rho \cosh \zeta_{j} s .
$$

If $\Omega_{j} \notin I(j ; s ; \delta)$, then

$$
\left|m \pi+\Theta\left(-\frac{\pi}{4} ; \frac{1}{\epsilon_{j}}\right)-\Omega_{j}\right| \geq \frac{\delta}{\sqrt{s}}, \quad \forall m \in \mathbb{Z},
$$

and, with Lemma 2.3 (e) and (f), we can conclude that

$$
\left|\Theta\left(m \pi+\Theta\left(-\frac{\pi}{4} ; \frac{1}{\epsilon_{j}}\right), \epsilon_{j}\right)-\Theta\left(\Omega_{j} ; \epsilon_{j}\right)\right| \geq \frac{c_{1} \delta}{\sqrt{s}} ;
$$

so, by Lemma 2.3 (c),

$$
\left|m \pi-\left(\frac{\pi}{4}+\Theta\left(\Omega_{j} ; \epsilon_{j}\right)\right)\right| \geq \frac{c_{1} \delta}{\sqrt{s}}, \quad \forall m \in \mathbb{Z}
$$

where $c_{1}>0$ depends only on $\epsilon_{j}$. Hence

$$
\sqrt{2}\left|\sin \left(\frac{\pi}{4}+\Theta\left(\Omega_{j} ; \epsilon_{j}\right)\right)\right| \geq \frac{c_{1} \delta}{\sqrt{s}}
$$

for all $s>\left(\frac{4 c_{1} \delta}{\pi}\right)^{2}$. Consequently, for all such $s$,

$$
\left|\sin \Theta\left(\Omega_{j} ; \epsilon_{j}\right)+\cos \Theta\left(\Omega_{j} ; \epsilon_{j}\right)\right| \geq \frac{c_{1} \delta}{\sqrt{s}}
$$

, which enables us to conclude that

$$
\frac{s y\left(a_{j+1}\right)}{y^{\prime}\left(a_{j+1}\right)}=\nu_{j}\left(1+O\left(\frac{1}{\sqrt{s}}\right)\right)
$$

and (2.4) is an immediate consequence.

From Theorem 2.6 and Lemma 2.5 we obtain the following corollary, which illustrates the behaviour of the modified Prüfer angle at the right end of an interval of negative weight, as a function of the modified Prüfer angle at the left end of the interval.

Corollary 2.7. Let the modified Prüfer angle at $a_{j}$ be $\Omega_{j}, j=0, \ldots, n$. Suppose that $r<c<0$ on $\left(a_{j}, a_{j+1}\right)$, where $c$ is a constant. Let $\delta>0$. Then, for large $s$, $\Omega_{j+1}$ is a continuous strictly increasing function of $\Omega_{j}$, such that if $\Omega_{j}$ is increased by $k \pi, k \in \mathbb{Z}$, then $\Omega_{j+1}$ increases by $k \pi$ and the point $\left(\Omega_{j}, \Omega_{j+1}\right)$ in $\mathbb{R}^{2}$ satisfies the relation

$$
\left(\Omega_{j}, \Omega_{j+1}\right) \in \bigcup_{m \in \mathbb{Z}}\left[\left[I_{m}^{\prime}(j ; s ; \delta) \times J_{m}(j ; s ; \delta)\right] \cup\left[I_{m+1}(j ; s ; \delta) \times J_{m}^{\prime}(j ; s ; \delta)\right]\right],
$$

where

$$
\begin{aligned}
& I_{m}^{\prime}(j ; s ; \delta)=\left[m \pi+\Theta\left(-\frac{\pi}{4} ; \frac{1}{\epsilon_{j}}\right)+\frac{\delta}{\sqrt{s}},(m+1) \pi+\Theta\left(-\frac{\pi}{4} ; \frac{1}{\epsilon_{j}}\right)-\frac{\delta}{\sqrt{s}}\right], \\
& J_{m}^{\prime}(j ; s ; \delta)=\left(m \pi+\Theta\left(\frac{\pi}{4} ; \nu_{j}\right)-\frac{L_{j}(\delta)}{\sqrt{s}},(m+1) \pi+\Theta\left(\frac{\pi}{4} ; \nu_{j}\right)+\frac{L_{j}(\delta)}{\sqrt{s}}\right),
\end{aligned}
$$

and $I_{m}(j ; s ; \delta), J_{m}(j ; s ; \delta)$ and $L_{j}(\delta)>0$ are as in Theorem 2.6. 
Proof. After consideration of Theorem [2.6] it is evident that all that is needed to prove this corollary is to match up the $I_{m}$ and $J_{r}$ indices. In order to perform this matching it is sufficient to obtain the pairing at some single point on the $\left(\Omega_{j} ; \Omega_{j+1}\right)$ curve, after which all other matchings are uniquely determined by periodicity and monotonicity of the curve.

We consider the case of $\Omega_{j}=\pi / 4$. In this case $y\left(a_{j}\right), y^{\prime}\left(a_{j}\right)>0$. Let $x$ be the minimal value (if there is one) in $\left[a_{j}, a_{j+1}\right]$ such that $y(x) y^{\prime}(x)=0$. If $y(x)=0$, then $y^{\prime}(t)>0$ for all $t \in\left(a_{j}, x\right)$, so $y(x)>y\left(a_{j}\right)>0$, contradiction. Thus $y(t)>0$ for all $t \in\left[a_{j}, x\right]$, and by continuity $y(t)>b>0$, say. It follows that $y^{\prime}(x)-y^{\prime}\left(a_{j}\right)=\int_{a_{j}}^{x}\left(q-s^{2} r\right) y>b \int_{a_{j}}^{x}\left(q+s^{2}|c|\right)>0$ for $s^{2}>|c|^{-1}\|q\|_{1}$, whence $y^{\prime}(x)>y^{\prime}\left(a_{j}\right)>0$. This second contradiction shows that $x$ does not exist for large $s$, and so the corresponding $\Omega_{j+1}$ is in the interval $(0, \pi / 2)$.

Thus we have determined that $\Omega_{j} \in I_{0}^{\prime}(j ; s ; \delta)$ gives $\Omega_{j+1} \in J_{0}(j ; s ; \delta)$. But the monotonicity of $\Omega_{j+1}$ as a function of $\Omega_{j}$ then shows that if $\Omega_{j} \in I_{1}(j ; s ; \delta)$, then $\Omega_{j+1} \in J_{0}^{\prime}(j ; s ; \delta)$. The periodicity of the same function completes the proof.

Corollary 2.7 can be illustrated diagrammatically by the graph of $\Omega_{j+1}\left(\Omega_{j}\right)$ as in Figure 1.

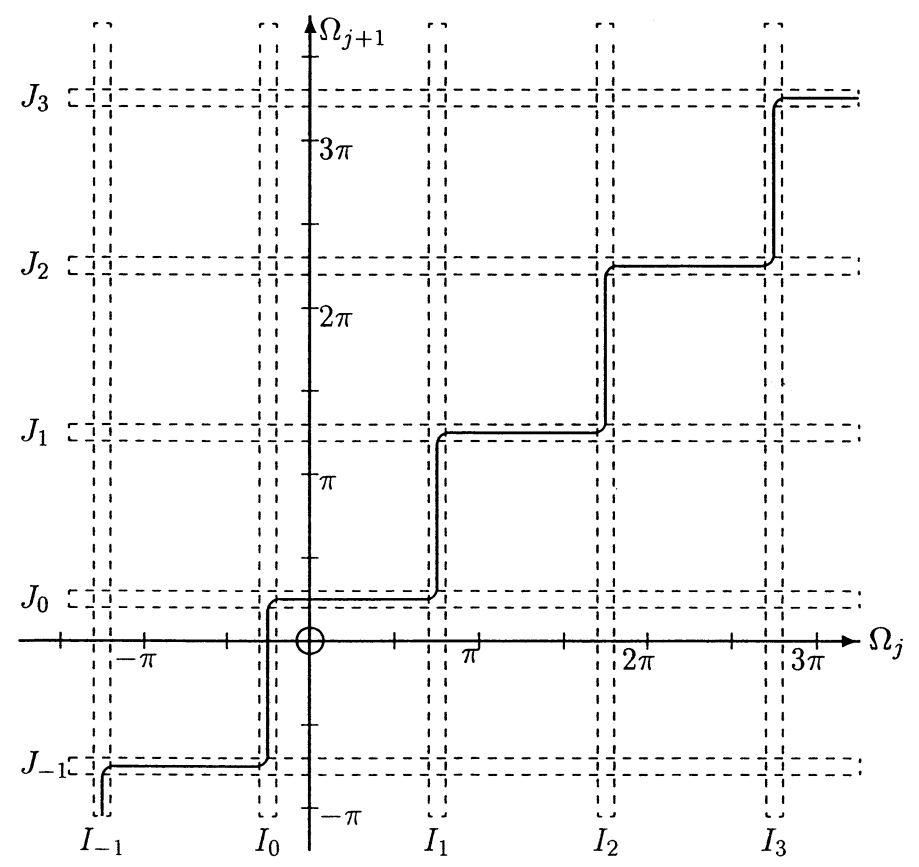

Figure $1 . \Omega_{j+1}$ as a function of $\Omega_{j}$

We end this section by giving an expression for the modified Prüfer angle $\Omega_{j+2}$ at $a_{j+2}$ in terms of the modified Prüfer angle $\Omega_{j}$ at $a_{j}$, under the condition that $r$ is positive on the interval $\left(a_{j}, a_{j+1}\right)$ and hence negative on the interval $\left(a_{j+1}, a_{j+2}\right)$. This is a direct consequence of (2.3) and Corollary [2.7.

Corollary 2.8. Suppose that $0 \leq j \leq n-2, r>c>0$ on $\left(a_{j}, a_{j+1}\right)$, and $r<$ $-c<0$ on $\left(a_{j+1}, a_{j+2}\right)$, where $c$ is a constant. Then $\Omega_{j+2}=\Omega\left(a_{j+2}, s, a_{j}, \Omega_{j}\right)$ 
is a continuous strictly increasing function of $\Omega_{j}$, such that if $\Omega_{j}$ is increased by $k \pi, k \in \mathbb{Z}$, then $\Omega_{j+2}$ increases by $k \pi$, and for large $s$ we have

$$
\begin{aligned}
\left(\zeta_{j} s\right. & \left.+\Theta\left(\Omega_{j} ; \epsilon_{j}\right) ; \Omega_{j+2}\right) \\
& \in \bigcup_{m \in \mathbb{Z}}\left[\left[\tilde{I}_{m}^{\prime}(j ; s ; \delta) \times \tilde{J}_{m}(j ; s ; \delta)\right] \cup\left[\tilde{I}_{m+1}(j ; s ; \delta) \times \tilde{J}_{m}^{\prime}(j ; s ; \delta)\right]\right],
\end{aligned}
$$

where

$$
\begin{aligned}
\tilde{I}_{m}(j ; s ; \delta) & =\left(m \pi+\Theta\left(-\frac{\pi}{4} ; \rho_{j}\right)-\frac{\delta}{\sqrt{s}}, m \pi+\Theta\left(-\frac{\pi}{4} ; \rho_{j}\right)+\frac{\delta}{\sqrt{s}}\right), \\
\tilde{I}_{m}^{\prime}(j ; s ; \delta) & =\left[m \pi+\Theta\left(-\frac{\pi}{4} ; \rho_{j}\right)+\frac{\delta}{\sqrt{s}},(m+1) \pi+\Theta\left(-\frac{\pi}{4} ; \rho_{j}\right)-\frac{\delta}{\sqrt{s}}\right], \\
\tilde{J}_{m}(j ; s ; \delta) & =\left[m \pi+\Theta\left(\frac{\pi}{4} ; \nu_{j+1}\right)-\frac{\tilde{L}_{j}(\delta)}{\sqrt{s}}, m \pi+\Theta\left(\frac{\pi}{4} ; \nu_{j+1}\right)+\frac{\tilde{L}_{j}(\delta)}{\sqrt{s}}\right], \\
\tilde{J}_{m}^{\prime}(j ; s ; \delta) & =\left(m \pi+\Theta\left(\frac{\pi}{4} ; \nu_{j+1}\right)-\frac{\tilde{L}_{j}(\delta)}{\sqrt{s}},(m+1) \pi+\Theta\left(\frac{\pi}{4} ; \nu_{j+1}\right)+\frac{\tilde{L}_{j}(\delta)}{\sqrt{s}}\right),
\end{aligned}
$$

and $\tilde{L}_{j}>0$ is a constant independent of $s$ and $\Omega_{j}$.

The resultant behaviour of $\Omega_{j+2}$ as a function of $\Omega_{j}$ and $s$ is illustrated in Figure 2. Note that the horizontal axis refers to $\zeta_{j} s+\Theta\left(\Omega_{j} ; \epsilon_{j}\right)$.

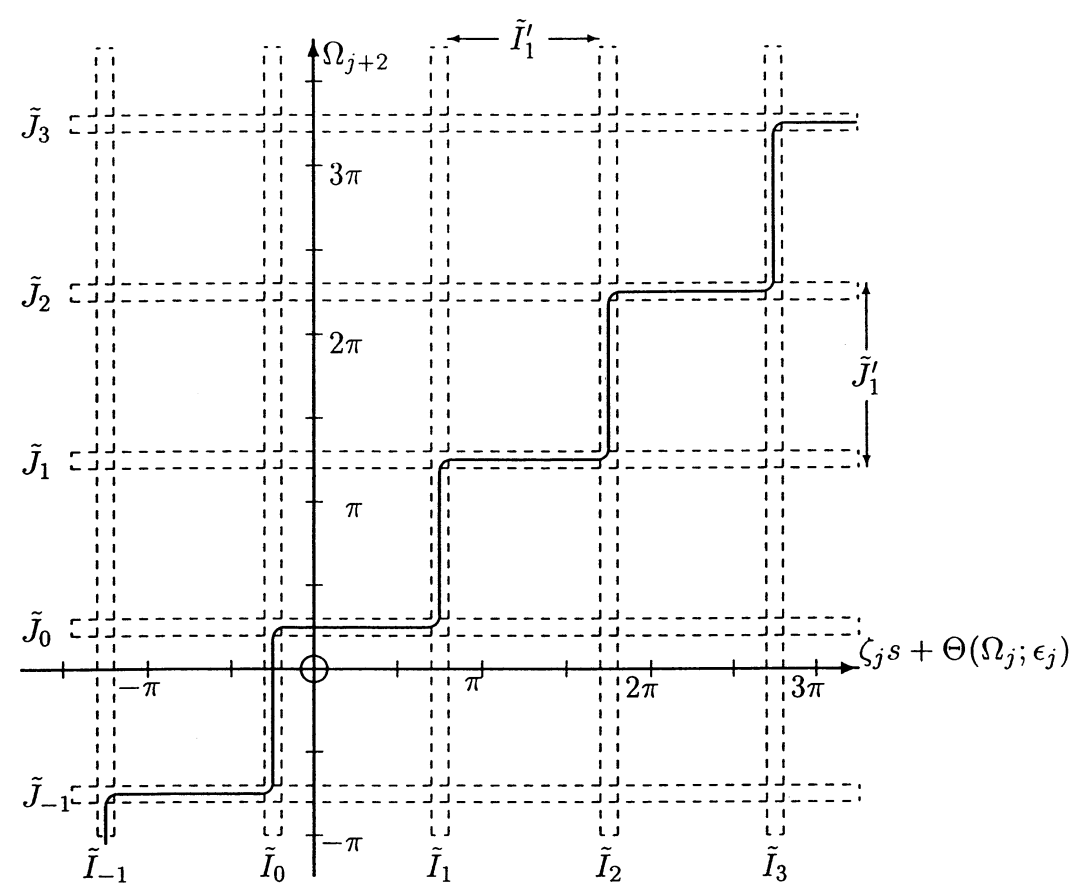

FiguRE 2. $\Omega_{j+2}$ as a function of $\zeta_{j} s+\Theta\left(\Omega_{j} ; \epsilon_{j}\right)$ 


\section{EigenVAlue PROBlEMS WITH $s$-DEPENDENT BOUNDARY CONDITIONS}

In this section we shall discuss asympotics on subintervals. Before discussing eigenvalues, we build on the results of Section 2 by considering $\Omega$ at one end of an interval given its value at the other end. As we shall see, this involves $s$-dependent conditions at the $a_{j}, 0<j<n$. We start with $r<0$ on the first subinterval, $\Omega$ being specified at $a_{0}=0$ via (1.2), i.e.,

$$
\Omega_{0}=\Theta(\alpha ; s) .
$$

Lemma 3.1. If (3.1) holds, then

$$
\Omega_{0}= \begin{cases}-\frac{\pi}{2}+O(1 / s), & \alpha \in(-\pi / 2,0), \\ 0, & \alpha=0, \\ \frac{\pi}{2}+O(1 / s), & \alpha \in(0, \pi / 2]\end{cases}
$$

and if, in addition, $r<c<0$ on $\left(a_{0}, a_{1}\right)$, then

$$
\Omega_{1}=\Omega\left(a_{1}, s, 0, \Omega_{0}\right)= \begin{cases}\tan ^{-1} \nu_{0}+O\left(\frac{1}{\sqrt{s}}\right), & \alpha \in[0, \pi / 2], \\ -\pi+\tan ^{-1} \nu_{0}+O\left(\frac{1}{\sqrt{s}}\right), & \alpha \in(-\pi / 2,0) .\end{cases}
$$

Here and below, $\cot ^{-1}$ and $\tan ^{-1}$ are assumed to take values in the range $(-\pi / 2, \pi / 2]$.

Proof. The expression for $\Omega_{0}$ follows from (3.1). Referring to Corollary 2.7 (see Fig. 1), we see that $\Omega_{1} \in J_{-1}$ in the first case and $\Omega_{1} \in J_{0}$ in the other two.

For the case $r>0$ on the final interval $\left(a_{n-1}, a_{n}\right)$ we will also denote the modified Prüfer angle there by $\Phi$ instead of $\Omega$. In particular, (1.3) implies

$$
\Phi_{n}=\Theta(\beta ; s)
$$

at $a_{n}$, and the corresponding $\Phi_{n-1}$ at $a_{n-1}$ obeys the following estimates.

Lemma 3.2. If (3.2) holds and $r>c>0$ on $\left(a_{n-1}, a_{n}\right)$, where $c$ is a constant, then

$$
\Phi_{n-1}=\Omega\left(a_{n-1}, s, l, \Phi_{n}\right)= \begin{cases}\Theta\left(-\zeta_{n-1} s-\frac{\pi}{2} ; \frac{1}{\epsilon_{n-1}}\right)+O\left(\frac{1}{s}\right), & \beta \in(-\pi / 2,0), \\ \Theta\left(-\zeta_{n-1} s ; \frac{1}{\epsilon_{n-1}}\right)+O\left(\frac{1}{s}\right), & \beta=0, \\ \Theta\left(-\zeta_{n-1} s+\frac{\pi}{2} ; \frac{1}{\epsilon_{n-1}}\right)+O\left(\frac{1}{s}\right), & \beta \in(0, \pi / 2] .\end{cases}
$$

Proof. It is easily verified that

$$
\Phi_{n}= \begin{cases}-\frac{\pi}{2}+O(1 / s), & \beta \in(-\pi / 2,0), \\ 0, & \beta=0, \\ \frac{\pi}{2}+O(1 / s), & \beta \in(0, \pi / 2] .\end{cases}
$$

From Theorem 2.6 (a) with $j=n-1$ and Lemma 2.3 (c) and (e) we have

$$
\Phi_{n-1}=\Theta\left(-\zeta_{n-1} s+\Theta\left(\Phi_{n} ; \frac{1}{\nu_{n-1}}\right) ; \frac{1}{\epsilon_{n-1}}\right)+O\left(\frac{1}{s}\right) .
$$

Combining the above with (3.4), we obtain (3.3).

Before proceeding we need a lemma concerning the $s$-derivative of $\Omega_{j+1}$ if $\Omega_{j}$ is specified (and vice versa). 
Lemma 3.3. Consider (1.1) on an interval $\left(a_{j}, a_{j+1}\right)$, with $r>c>0$.

(a) If $\Omega_{j+1}=\Omega\left(a_{j+1}, s, a_{j}, \Omega_{j}\right)$ for $j \leq n$, then there exist positive constants $c^{-}, c^{+}$such that for large $s$,

$$
c^{-} \leq \dot{\Omega}_{j+1} \leq c^{+}
$$

where ${ }^{\cdot}=\frac{\partial}{\partial s}$.

(b) If $\Omega_{j}=\Omega\left(a_{j}, s, a_{j+1}, \Omega_{j+1}\right)$ for $j \leq n-1$, then there exist positive constants $c^{-}, c^{+}$such that for large $s$,

$$
c^{-} \leq-\dot{\Omega}_{j} \leq c^{+} .
$$

Proof. For this proof we use the modified Prüfer angle of [2]. Let

$$
\psi=\Theta(\Omega ; \sqrt{r}),
$$

so $\tan \psi=s y \sqrt{r} / y^{\prime}$, where $y$ satisfies (1.1), and hence

$$
\psi^{\prime}=s \sqrt{r}-\frac{q}{s \sqrt{r}} \sin ^{2} \psi+\frac{r^{\prime}}{2 r} \sin \psi \cos \psi .
$$

Differentiating the above with respect to $s$, we get

$$
\dot{\psi}^{\prime}=\sqrt{r}+\frac{q}{s^{2} \sqrt{r}} \sin ^{2} \psi+\left[\frac{r^{\prime}}{2 r} \cos 2 \psi-\frac{q}{s \sqrt{r}} \sin 2 \psi\right] \dot{\psi} .
$$

Treating (3.7) as a first order linear equation in $\dot{\psi}$, we see that (3.7) has integrating factor

$$
h(t)=\exp \int_{t}^{a_{j+1}}\left[\frac{r^{\prime}}{2 r} \cos 2 \psi-\frac{q}{s \sqrt{r}} \sin 2 \psi\right] d \tau,
$$

and there exist $\gamma>0$ and $K>0$ such that $1 / \gamma \leq h(t) \leq \gamma$, for all $t \in\left[a_{j}, a_{j+1}\right]$ and $s>K$. Thus

$$
\dot{\psi}\left(a_{j+1}\right)-\dot{\psi} h\left(a_{j}\right)=\int_{a_{j}}^{a_{j+1}}\left(\sqrt{r}+\frac{q}{s^{2} \sqrt{r}} \sin ^{2} \psi\right) h d \tau,
$$

giving

$$
\left|\dot{\psi}\left(a_{j+1}\right)-\dot{\psi} h\left(a_{j}\right)-\int_{a_{j}}^{a_{j+1}} \sqrt{r} h d \tau\right| \leq \frac{\kappa \gamma}{s^{2}},
$$

where $\kappa=\int_{a_{j}}^{a_{j+1}} \frac{|q|}{\sqrt{r}} d \tau$.

In the case of (3.10), $\dot{\psi}\left(a_{j}\right)=0$, and (3.5) follows easily from (3.8).

In the case of (1.2),

$$
\dot{\psi}\left(a_{j}\right)=\frac{\tan \alpha}{1+s^{2} \tan ^{2} \alpha},
$$

which with (3.8) gives

$$
\left|\dot{\psi}\left(a_{j+1}\right)-\int_{a_{j}}^{a_{j+1}} \sqrt{r} h d \tau\right| \leq \frac{\kappa_{1} \gamma}{s^{2}}
$$

for some positive constant $\kappa_{1}$ which depends on $r, q$ and $\alpha$. From (3.9), (3.5) follows easily.

Dividing (3.8) by $h\left(a_{j}\right)$, we can reason as above to establish (3.6). 
Since the eigenvalues are ordered by Sturm's Theorem, we shall also need the following elementary result on oscillation counts. When we consider oscillation counts, the modified Prüfer angle and the standard Prüfer angle behave in a similar manner, enabling us to deduce the next result as in the case of the unmodified Prüfer angle.

Lemma 3.4. If $\omega \in(-\pi / 2, \pi / 2]$ and $\Omega(b ; s ; a ; \omega)=B+m \pi$, where $m \in \mathbb{N}, B \in$ $(-\pi / 2, \pi / 2]$, then the oscillation count across $(a, b)$ of non-trivial solutions of (1.1) is

$$
\begin{aligned}
m+1 & \text { if } \omega<0 \quad \text { and } \quad B>0 \\
m & \text { if } \omega \geq 0 \quad \text { and } \quad B>0 \\
m & \text { if } \omega<0 \quad \text { and } B \leq 0, \\
m-1 & \text { if } \omega \geq 0 \quad \text { and } B \leq 0 \quad(m>0) .
\end{aligned}
$$

We are now ready for the main result of this section, which gives eigenvalue asymptotics for Sturm-Liouville problems with $s$-dependent boundary conditions on subintervals where $r>0$. Note that $\Omega_{0}$ is specified via (3.1), $\Omega_{n}$ by $\Theta(\beta ; s)$ (cf. $(\overline{3.2})$ ), and for $0<j<n$ the specification of $\Omega_{j}$ is equivalent to the $s$-dependent condition

$$
s y\left(a_{j}\right)=\left(\tan \Omega_{j}\right) y^{\prime}\left(a_{j}\right)
$$

on $(1.1)$.

Theorem 3.5. On an interval $\left(a_{j}, a_{j+1}\right)$ where $r>c>0$, consider the equation (1.1) with boundary conditions of the form

$$
s y\left(a_{j}\right)=k^{+} y^{\prime}\left(a_{j}\right), \quad k^{+}>0
$$

for $j \geq 1$, and (1.2) for $j=0$, at $a_{j}$; and of the form

$$
\operatorname{sy}\left(a_{j+1}\right)=k^{-} y^{\prime}\left(a_{j+1}\right), \quad k^{-}<0,
$$

for $j+1 \leq n-1$, and (1.3) for $j+1=n$, at $a_{j+1}$. For each large $m \in \mathbb{N}$, this eigenvalue problem has one and only one eigenvalue $s=s_{m}>0$, which corresponds to an eigenfunction with oscillation count $m$. In addition, $s_{m}$ is given asymptotically by:

$\zeta_{j} s_{m}=\left\{\begin{array}{lll}\left(m+\frac{1}{2}\right) \pi-\tan ^{-1} k^{+} \epsilon_{n-1}+O\left(\frac{1}{m}\right), & j=n-1, & \beta \neq 0, \\ (m+1) \pi-\tan ^{-1} k^{+} \epsilon_{n-1}+O\left(\frac{1}{m}\right), & j=n-1, & \beta=0, \\ (m+1) \pi-\tan ^{-1} k^{+} \epsilon_{j}+\tan ^{-1}\left(\frac{k^{-}}{\nu_{j}}\right)+O\left(\frac{1}{m}\right), & 1 \leq j \leq n-2, & \\ \left(m+\frac{1}{2}\right) \pi+\tan ^{-1}\left(\frac{k^{-}}{\nu_{j}}\right)+O\left(\frac{1}{m}\right), & j=0, & \alpha \neq 0, \\ (m+1) \pi+\tan ^{-1}\left(\frac{k^{-}}{\nu_{j}}\right)+O\left(\frac{1}{m}\right), & j=0, & \alpha=0 .\end{array}\right.$

Proof. Let $\Omega_{j} \in(-\pi / 2, \pi / 2]$, the modified Prüfer angle at $a_{j}$, be given by $\tan \Omega_{j}=$ $k^{+}$for $j \neq 0$, and by (3.1) for $j=0$. Then, by Lemma 3.3, there exist $c^{-}, c^{+}>0$ such that (3.5) holds for large $s$.

The eigenvalue condition at $a_{j+1}$ for $j+1=n$ becomes

$$
\Omega_{n}=\Theta(\beta ; s)+m \pi, \quad m \in \mathbb{Z},
$$

and the $s$-derivative of the right-hand side of the above equation is $O\left(1 / s^{2}\right)$. Along with (3.5) this shows that for each large $m \in \mathbb{N}$ there is one and only one $s>0$ solving (3.13). 
The eigenvalue condition at $a_{j+1}$ for $j+1 \leq n-1$ becomes

$$
\Omega_{j+1}=\tan ^{-1} k^{-}+m \pi, \quad m \in \mathbb{Z},
$$

and the $s$-derivative of the right hand side of the above equation is zero. Along with (3.5) this gives that for each large $m \in \mathbb{N}$ there is one and only one $s>0$ solving (3.13).

The oscillation count corresponding to each $m$ can be determined from Lemma 3.4.

It remains to give an asymptotic expression for the sequence $s_{m}, m \in \mathbb{N}$.

For the case $j=n-1$, Theorem 2.6 (a) and (3.13) give

$$
\zeta_{n-1} s=-\Theta\left(\Omega_{n-1} ; \epsilon_{n-1}\right)+\tan ^{-1}\left(\frac{s \tan \beta}{\nu_{n-1}}\right)+m \pi+O(1 / m) .
$$

Since $n \geq 2$, (3.11) gives $\Omega_{n-1}=\tan ^{-1} k^{+}$, and so

$$
\zeta_{n-1} s=-\tan ^{-1}\left(k^{+} \epsilon_{n-1}\right)+\tan ^{-1}\left(\frac{s \tan \beta}{\nu_{n-1}}\right)+m \pi+O(1 / m)
$$

and from Lemma 3.4 the oscillation count corresponding to $s$ is $m$ if $\beta \in(0, \pi / 2]$ and $m-1$ for $\beta \in(-\pi / 2,0]$.

In the case $1 \leq j \leq n-2$, Theorem 2.6 (a), 3.11) and (3.14) give

$$
\zeta_{j} s=-\tan ^{-1}\left(k^{+} \epsilon_{j}\right)+\tan ^{-1}\left(\frac{k^{-}}{\nu_{j}}\right)+m \pi+O(1 / m),
$$

and from Lemma 3.4 the oscillation count corresponding to $s$ is $m-1$.

In the case $j=0$, (3.1) and (3.14) give

$$
\zeta_{j} s=-\tan ^{-1}\left(s \epsilon_{j} \tan \alpha\right)+\tan ^{-1}\left(\frac{k^{-}}{\nu_{j}}\right)+m \pi+O(1 / m),
$$

and from Lemma 3.4 the oscillation count corresponding to $s$ is $m-1$ if $\alpha \in[0, \pi / 2]$ and $m$ for $\alpha \in(-\pi / 2,0)$.

Corollary 3.6. If $r>0$ on $\left(a_{j}, a_{j+1}\right)$, then $\sigma_{j}$ (see Theorem 1.2) is given asymptotically by

$\zeta_{j} s_{m j}=\left\{\begin{array}{lll}\left(m+\frac{1}{2}\right) \pi-\cot ^{-1} \rho_{j-1}+O\left(\frac{1}{m}\right), & j=n-1, & \beta \neq 0, \\ (m+1) \pi-\cot ^{-1} \rho_{j-1}+O\left(\frac{1}{m}\right), & j=n-1, & \beta=0, \\ (m+1) \pi-\cot ^{-1} \rho_{j-1}-\tan ^{-1} \rho_{j}+O\left(\frac{1}{m}\right), & 1 \leq j \leq n-2, & \\ \left(m+\frac{1}{2}\right) \pi-\tan ^{-1} \rho_{j}+O\left(\frac{1}{m}\right), & j=0, & \alpha \neq 0, \\ (m+1) \pi-\tan ^{-1} \rho_{j}+O\left(\frac{1}{m}\right), & j=0, & \alpha=0,\end{array}\right.$

where $s_{m j}$ corresponds to an eigenfunction with $m$ zeros in $(0, l)$.

Proof. Comparing (1.9) and (1.10) with (3.11) and (3.12), we see that $k^{+}=\nu_{j-1}^{-1}$ and $k^{-}=-\epsilon_{j+1}^{-1}$. The result now follows from Theorem 3.5

\section{The MOdified Prüfer ANGLE}

In this section two lemmata are given which express the modified Prüfer angle, as a function of $s$, at the right-hand end of a subinterval where $r$ is negative, under the assumption that the modified Prüfer angle $\Omega_{0}$ at $a_{0}=0$ is given by (3.1). See Corollary 2.8 and Figure 2 for the notation $\tilde{J}$ and $\tilde{J}^{\prime}$ below. 
Lemma 4.1. Let $r>c>0$ on $\left(a_{2 j}, a_{2 j+1}\right), j=0, \ldots, k$, and $r<-c<0$ on $\left(a_{2 j+1}, a_{2 j+2}\right), j=0, \ldots, k$. Let $s_{0} \leq s_{1} \leq s_{2} \leq \ldots$ be a listing of

$$
\Sigma=\bigcup_{i=0, \ldots, 2 k+1}^{\bullet} \sigma_{i}
$$

(so each element of $\dot{\bigcup} \sigma_{i}$ is repeated according to its multiplicity). There exist constants $k_{1}, k_{2} \in \mathbb{Z}$ and $k_{0}, k_{3}>0$ such that if $m \geq k_{1}$, then

$$
s \in P_{m} \quad \Longrightarrow \quad \Omega_{2 k+2} \in \tilde{J}_{m+k_{2}}\left(2 k, s, k_{3}\right) \text {, }
$$

where

$$
P_{m}=\left[s_{m}+\frac{k_{0}}{\sqrt{s_{m}}}, s_{m+1}-\frac{k_{0}}{\sqrt{s_{m+1}}}\right],
$$

and, for $m$ such that $P_{m-1} \neq \emptyset$ and integers $N \geq 0$ such that $P_{m+N} \neq \emptyset$,

$$
s \in\left(s_{m}-\frac{k_{0}}{\sqrt{s_{m}}}, s_{m+N}+\frac{k_{0}}{\sqrt{s_{m+N}}}\right) \Longrightarrow \Omega_{2 k+2} \in \bigcup_{i=m}^{m+N} \tilde{J}_{i-1+k_{2}}^{\prime}\left(2 k, s, k_{3}\right),
$$

where $\Omega_{2 k+2}=\Omega\left(a_{2 k+2}, s, 0, \Omega_{0}\right)$.

Proof. For brevity we will present the proof only for the case of $\alpha \in(0, \pi / 2]$; the proofs for $\alpha=0$ and $\alpha \in(-\pi / 2,0)$ are similar to the case presented.

(i) We proceed by induction on $k$. For $k=0$ let $k_{0}=4 / \zeta_{0}, k_{2}=1$ and $k_{3}=1$. Our first step is to show that

$$
s \in P_{m}=\left[s_{m}+\frac{4}{\zeta_{0} \sqrt{s_{m}}}, s_{m+1}-\frac{4}{\zeta_{0} \sqrt{s_{m+1}}}\right] \quad \Longrightarrow \quad \Omega_{2} \in \tilde{J}_{m+1}(0, s, 1) .
$$

From Lemmas 3.1 and 2.3 we have

$$
\Theta\left(\Omega_{0}(s) ; \epsilon_{0}\right)-\frac{\pi}{2} \geq-\frac{1}{\sqrt{s}},
$$

for sufficiently large $s$. Now Corollary 3.6 gives

$$
\left|\zeta_{0} s_{i}+\tan ^{-1} \rho_{0}-\left(i+\frac{1}{2}\right) \pi\right|<\frac{1}{\sqrt{s_{i}}},
$$

for sufficiently large $i$. Combining (4.3), with $i=m$, and (4.2) for $s \geq s_{m}+\frac{4}{\zeta_{0} \sqrt{s_{m}}}$, we obtain

$$
\begin{aligned}
\zeta_{0} s & +\Theta\left(\Omega_{0}(s) ; \epsilon_{0}\right)-\frac{\pi}{2} \\
& \geq \zeta_{0} s_{m}+\frac{4}{\sqrt{s_{m}}}-\frac{1}{\sqrt{s}} \\
& >\left(m+\frac{1}{2}\right) \pi-\tan ^{-1} \rho_{0}-\frac{1}{\sqrt{s_{m}}}+\frac{4}{\sqrt{s_{m}}}-\frac{1}{\sqrt{s}} \\
& \geq\left(m+\frac{1}{2}\right) \pi-\tan ^{-1} \rho_{0}+\frac{2}{\sqrt{s}} .
\end{aligned}
$$

Hence for sufficiently large $m$ we have

$$
\zeta_{0} s+\Theta\left(\Omega_{0}(s) ; \epsilon_{0}\right)>(m+1) \pi-\tan ^{-1} \rho_{0}+\frac{1}{\sqrt{s}} .
$$


Since $\alpha \in(0, \pi / 2]$, we have

$$
\Theta\left(\Omega_{0}(s) ; \epsilon_{0}\right)-\frac{\pi}{2} \leq 0 .
$$

Combining (4.3), with $i=m+1$, (4.5) and $s \leq s_{m+1}-\frac{4}{\zeta_{0} \sqrt{s_{m+1}}}$, we obtain

$$
\begin{aligned}
\zeta_{0} s & +\Theta\left(\Omega_{0}(s) ; \epsilon_{0}\right)-\frac{\pi}{2} \leq \zeta_{0} s_{m+1}-\frac{4}{\sqrt{s_{m+1}}} \\
& <\left(m+\frac{3}{2}\right) \pi-\tan ^{-1} \rho_{0}-\frac{3}{\sqrt{s_{m+1}}} .
\end{aligned}
$$

Now for large $m$, we have $s_{m+1} \leq 9 s_{m} \leq 9 s$; so

$$
\zeta_{0} s+\Theta\left(\Omega_{0}(s) ; \epsilon_{0}\right)<(m+2) \pi-\tan ^{-1} \rho_{0}-\frac{1}{\sqrt{s}} .
$$

Considering both (4.4) and (4.6), we see that $\zeta_{0} s+\Theta\left(\Omega_{0}(s) ; \epsilon_{0}\right) \in \tilde{I}_{m+1}^{\prime}(0 ; s ; 1)$. Thus from Corollary 2.8 we have $\Omega_{2}(s) \in \tilde{J}_{m+1}(0 ; s ; 1)$, which proves 4.1) for $k=0$.

(ii) We next demonstrate

$$
s \in Q:=\left(s_{m}-\frac{4}{\zeta_{0} \sqrt{s_{m}}}, s_{m+N}+\frac{4}{\zeta_{0 \sqrt{s_{m+N}}}}\right) \quad \Longrightarrow \quad \Omega_{2} \in \bigcup_{i=m}^{m+N} \tilde{J}_{i}^{\prime}(0, s, 1),
$$

assuming $P_{m-1}$ and $P_{m+N}$ are nonempty (which is automatic for $k=0$ and sufficiently large $m$, since $\Sigma=\sigma_{0}$ consists of simple eigenvalues).

For $s \geq s_{m-1}+\frac{4}{\zeta_{0} \sqrt{s_{m-1}}}$ we have

$$
\zeta_{0} s+\Theta\left(\Omega_{0}(s) ; \epsilon_{0}\right)-m \pi+\tan ^{-1} \rho_{0}>\frac{1}{\sqrt{s}},
$$

as for (4.4) but with $m$ replaced by $m-1$. For $s \leq s_{m+N+1}-\frac{4}{\zeta_{0} \sqrt{s_{m+N+1}}}$,

$$
\zeta_{0} s+\Theta\left(\Omega_{0}(s) ; \epsilon_{0}\right)-(m+N+2) \pi+\tan ^{-1} \rho_{0}<-\frac{1}{\sqrt{s}},
$$

as for (4.6) but with $m$ replaced by $m+N$.

Considering both (4.7) and (4.8), we see that for $s \in Q$,

$$
\frac{1}{\sqrt{s}}+m \pi<\zeta_{0} s+\Theta\left(\Omega_{0}(s) ; \epsilon_{0}\right)+\tan ^{-1} \rho_{0}<(m+N+2) \pi-\frac{1}{\sqrt{s}},
$$

and thus from Corollary 2.8 we have

$$
m \pi+\tan ^{-1} \nu_{1}-\frac{\tilde{L}_{0}(1)}{\sqrt{s}}<\Omega_{2}(s)<(m+N+1) \pi+\tan ^{-1} \nu_{1}+\frac{\tilde{L}_{0}(1)}{\sqrt{s}} .
$$

This completes the proof of (ii) and hence of the case $k=0$.

(iii) We now proceed to the induction step, and assume the lemma to be true for $k \geq 0$. Let $s_{0} \leq s_{1} \leq s_{2} \leq \ldots$ be a listing of

$$
\Sigma=\bigcup_{i=0, \ldots, 2 k+1}^{\bullet} \sigma_{i}
$$

where each element is repeated according to its multiplicity; let $\tilde{s}_{0} \leq \tilde{s}_{1} \leq \tilde{s}_{2} \leq \ldots$ be a similar listing of

$$
\tilde{\Sigma}=\bigcup_{i=0, \ldots, 2 k+3}^{\bullet} \sigma_{i}
$$


(so $\tilde{\Sigma}=\Sigma \dot{U} \sigma_{2 k+2}$ since $\sigma_{2 k+3}=\emptyset$ ), and finally let $\eta_{0}<\eta_{1}<\ldots$ denote the elements of $\sigma_{2 k+2}$.

From the assumptions on the induction step we see that $\Omega_{2 k+2}$, as a function of $s$, is of the form illustrated in Figure 3.

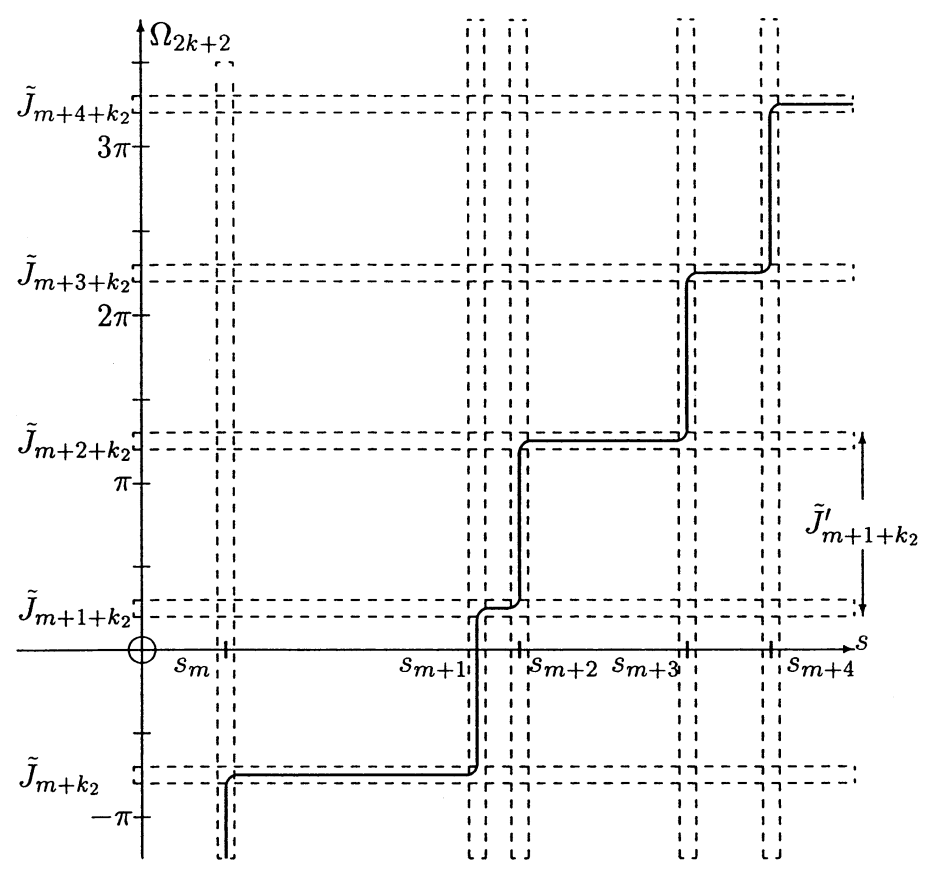

Figure $3 . \Omega_{2 k+2}$ as a function of $s$

(iv) We now show there are constants $\tilde{k}_{0}, \tilde{k}_{3}>0$ such that for each large $m$

$$
s \in \tilde{P}_{m} \Longrightarrow \Omega_{2 k+4} \in \tilde{J}_{m+\tilde{k}_{2}}\left(2 k+2, s, \tilde{k}_{3}\right),
$$

for some integer $\tilde{k}_{2}=\tilde{k}_{2}(m)$, where $\tilde{P}_{m}=\left[\tilde{s}_{m}+\frac{\tilde{k}_{0}}{\sqrt{\tilde{s}_{m}}}, \tilde{s}_{m+1}-\frac{\tilde{k}_{0}}{\sqrt{\tilde{s}_{m+1}}}\right]$. Denote by $M(\tau)$ the maximum of the set $\left\{\frac{\partial \Theta(\omega ; \tau)}{\partial \omega} ; \omega \in[0, \pi]\right\}$, cf. Lemma 2.3. Let $\tilde{k}_{3}=$ $\tilde{L}_{2 k}\left(k_{3}\right) M\left(\epsilon_{2 k+2}\right)$ and $\tilde{k}_{0}=2 k_{0}+\frac{3 \tilde{k}_{3}}{\zeta_{2 k+2}}$, where $\tilde{L}_{2 k}(\cdot)$ is as defined in Corollary 2.8 and $k_{0}, k_{2}$ and $k_{3}$ are given by the induction hypothesis. Let $d$ be the least $j$ such that $s_{j} \geq \tilde{s}_{m+1}$, and let $h$ be the least $j$ such that $\eta_{j} \geq \tilde{s}_{m+1}$. It is easily seen that $d$ and $h$ tend to infinity with $m$.

Note that for large enough $m$ (and hence $d$ ) we have $s_{d} / s_{d-1}<4$, and so $k_{0} / \sqrt{s_{d-1}}<\tilde{k}_{0} / \sqrt{\tilde{s}_{m}}$. It follows that $\tilde{P}_{m} \subset P_{d-1}$; so from the induction hypothesis we have $\Omega_{2 k+2}(s) \in \tilde{J}_{d-1+k_{2}}\left(2 k, s, k_{3}\right)$ for $s \in \tilde{P}_{m}$. Thus

$$
\Theta\left(\Omega_{2 k+2}(s) ; \epsilon_{2 k+2}\right)-\left(d+k_{2}-1\right) \pi-\cot ^{-1} \rho_{2 k+1} \geq-\frac{\tilde{k}_{3}}{\sqrt{s}}
$$

and

$$
\Theta\left(\Omega_{2 k+2}(s) ; \epsilon_{2 k+2}\right)-\left(d+k_{2}-1\right) \pi-\cot ^{-1} \rho_{2 k+1} \leq \frac{\tilde{k}_{3}}{\sqrt{s}}
$$


follow from Lemma [2.3.

From Corollary [3.6] with $j=2 k+2$, there exists an integer $A$ such that for large $i$

$$
\left|\zeta_{2 k+2} \eta_{i}+\cot ^{-1} \rho_{2 k+1}+\tan ^{-1} \rho_{2 k+2}-(i+A) \pi\right|<\frac{\zeta_{2 k+2} k_{0}}{\sqrt{\eta_{i}}}
$$

cf. (4.3). Combining (4.11), with $i=h-1$, (4.9) and $s \geq \eta_{h-1}+\tilde{k}_{0} / \sqrt{\tilde{s}_{m}}$, we obtain

$$
\begin{aligned}
\zeta_{2 k+2} s & +\Theta\left(\Omega_{2 k+2}(s) ; \epsilon_{2 k+2}\right)-\left(d+k_{2}-1\right) \pi \\
& \geq \zeta_{2 k+2} \eta_{h-1}+\frac{\zeta_{2 k+2} \tilde{k}_{0}}{\sqrt{\tilde{s}_{m}}}+\cot ^{-1} \rho_{2 k+1}-\frac{\tilde{k}_{3}}{\sqrt{s}} \\
& >(h+A-1) \pi-\tan ^{-1} \rho_{2 k+2}-\frac{\zeta_{2 k+2} k_{0}}{\sqrt{\eta_{h-1}}}+\frac{\zeta_{2 k+2} \tilde{k}_{0}}{\sqrt{\tilde{s}_{m}}}-\frac{\tilde{k}_{3}}{\sqrt{s}} \\
& =(h+A-1) \pi-\tan ^{-1} \rho_{2 k+2}+\frac{2 \tilde{k}_{3}}{\sqrt{s}}+\zeta_{2 k+2} k_{0}\left(\frac{2}{\sqrt{s_{m}}}-\frac{1}{\sqrt{\eta_{h-1}}}\right) .
\end{aligned}
$$

Now for sufficiently large $m$ (and hence $h$ ), $\tilde{s}_{m} \leq \eta_{h} \leq 4 \eta_{h-1}$; so

$$
\zeta_{2 k+2} s+\Theta\left(\Omega_{2 k+2}(s) ; \epsilon_{2 k+2}\right)>\left(h+A+d+k_{2}-2\right) \pi-\tan ^{-1} \rho_{2 k+2}+\frac{\tilde{k}_{3}}{\sqrt{s}} .
$$

Combining (4.11) with $i=h$, (4.10) and $s \leq \eta_{h}-\frac{\tilde{k}_{0}}{\sqrt{\tilde{s}_{m+1}}}$, we obtain

$$
\begin{aligned}
\zeta_{2 k+2} s & +\Theta\left(\Omega_{2 k+2}(s) ; \epsilon_{2 k+2}\right)-\left(d+k_{2}-1\right) \pi \\
& \leq \zeta_{2 k+2} \eta_{h}-\frac{\zeta_{2 k+2} \tilde{k}_{0}}{\sqrt{s_{m+1}}}+\cot ^{-1} \rho_{2 k+1}+\frac{\tilde{k}_{3}}{\sqrt{s}} \\
& <(h+A) \pi-\tan ^{-1} \rho_{2 k+2}+\frac{\zeta_{2 k+2} k_{0}}{\sqrt{\eta_{h}}}-\frac{\zeta_{2 k+2} \tilde{k}_{0}}{\sqrt{\eta_{h}}}+\frac{\tilde{k}_{3}}{\sqrt{s}} \\
& <(h+A) \pi-\tan ^{-1} \rho_{2 k+2}-\tilde{k}_{3}\left(\frac{3}{\sqrt{\eta_{h}}}-\frac{1}{\sqrt{s}}\right) .
\end{aligned}
$$

For sufficiently large $h$ we have $\frac{4}{9} \eta_{h} \leq \eta_{h-1} \leq s$. Hence we obtain

$$
\zeta_{2 k+2} s+\Theta\left(\Omega_{2 k+2}(s) ; \epsilon_{2 k+2}\right)<\left(h+d+A+k_{2}-1\right) \pi-\tan ^{-1} \rho_{2 k+2}-\frac{\tilde{k}_{3}}{\sqrt{s}} .
$$

Considering both (4.12) and (4.13), we see that

$$
\zeta_{2 k+2} s+\Theta\left(\Omega_{2 k+2}(s) ; \epsilon_{2 k+2}\right) \in \tilde{I}_{d+h+k_{2}+A-2}^{\prime}\left(2 k+2 ; s ; \tilde{k}_{3}\right) .
$$

Thus from Corollary 2.8 we have

$$
\Omega_{2 k+4}(s) \in \tilde{J}_{m+\tilde{k}_{2}(m)}\left(2 k+2 ; s ; \tilde{k}_{3}\right),
$$

where

$$
m+\tilde{k}_{2}(m)=d+h+k_{2}+A-2 .
$$


(v) The next step is to show that $\tilde{k}_{2}(m)$ constructed as above is in fact independent of $m$. Suppose that there exist

$$
s \in\left[\tilde{s}_{m-1}+\frac{\tilde{k}_{0}}{\sqrt{\tilde{s}_{m-1}}}, \tilde{s}_{m}-\frac{\tilde{k}_{0}}{\sqrt{\tilde{s}_{m}}}\right]
$$

and

$$
t \in\left[\tilde{s}_{m+N}+\frac{\tilde{k}_{0}}{\sqrt{\widetilde{s}_{m+N}}}, \tilde{s}_{m+N+1}-\frac{\tilde{k}_{0}}{\sqrt{\tilde{s}_{m+N+1}}}\right],
$$

where $N$ is a nonnegative integer. The sequence $\tilde{s}_{m} \leq \ldots \leq \tilde{s}_{m+N}$ can be decomposed into the disjoint union of two subsequences (either, but not both, of which may be empty) $s_{d} \leq \ldots \leq s_{w-1}$ and $\eta_{h}<\ldots<\eta_{u-1}$. Here $d$ and $h$ are as in (iv), and $w$ and $u$ are the corresponding integers with $m$ replaced by $m+N$. The numbers of elements in the subsequences are then $w-d$ and $u-h$ respectively, and it is to be noted that

$$
N=w-d+u-h
$$

From (4.14) we have

$$
\Omega_{2 k+4}(s) \in \tilde{J}_{d+h+k_{2}+A-2}\left(2 k+2 ; s ; \tilde{k}_{3}\right),
$$

and from the analogue of (4.14) with $t$ instead of $s$ we have

$$
\Omega_{2 k+4}(t) \in \tilde{J}_{w+u+k_{2}+A-2}\left(2 k+2 ; t ; \tilde{k}_{3}\right) .
$$

Thus

$$
m+\tilde{k}_{2}(m)=d+h+k_{2}+A-2
$$

and

$$
m+N+\tilde{k}_{2}(m+N)=w+u+k_{2}+A-2=d+h+N+k_{2}+A-2
$$

from (4.15); so $\tilde{k}_{2}(m)=\tilde{k}_{2}(m+N)$, as required. This establishes the inductive step for (4.1).

(vi) The final step is to show that

$$
\begin{aligned}
s \in \tilde{Q} & :=\left(\tilde{s}_{m}-\frac{\tilde{k}_{0}}{\sqrt{\tilde{s}_{m}}}, \tilde{s}_{m+N}+\frac{\tilde{k}_{0}}{\sqrt{\tilde{s}_{m+N}}}\right) \\
& \Longrightarrow \Omega_{2 k+4} \in \bigcup_{i=m}^{m+N} \tilde{J}_{i-1+\tilde{k}_{2}}^{\prime}\left(2 k+2, s, \tilde{k}_{3}\right)
\end{aligned}
$$

for each large $m$ and each integer $N \geq 0$, subject to $\tilde{P}_{m-1}$ and $\tilde{P}_{m+N}$ being nonempty.

From (4.12), $s \geq \tilde{s}_{m-1}+\frac{\tilde{k}_{0}}{\sqrt{\tilde{s}_{m-1}}}$ gives

$$
\zeta_{2 k+2} s+\Theta\left(\Omega_{2 k+2}(s) ; \epsilon_{2 k+2}\right)-\left(d+h+k_{2}+A-3\right) \pi+\tan ^{-1} \rho_{2 k+2}>\frac{\tilde{k}_{3}}{\sqrt{s}},
$$


and, from (4.13) with $s \leq \tilde{s}_{m+N+1}-\frac{\tilde{k}_{0}}{\sqrt{\tilde{s}_{m+N+1}}}$,

$$
\zeta_{2 k+2} s+\Theta\left(\Omega_{2 k+2}(s) ; \epsilon_{2 k+2}\right)-\left(w+u-1+k_{2}+A\right) \pi+\tan ^{-1} \rho_{2 k+2}<-\frac{\tilde{k}_{3}}{\sqrt{s}} .
$$

From (4.15), (4.16) and (4.17) we see that, for $s \in \tilde{Q}$,

$$
\begin{aligned}
& \frac{\tilde{k}_{3}}{\sqrt{s}}+\left(d+h+k_{2}+A-3\right) \pi \\
& \quad<\zeta_{2 k+2} s+\Theta\left(\Omega_{2 k+2}(s) ; \epsilon_{2 k+2}\right)+\tan ^{-1} \rho_{2 k+2} \\
& \quad<\left(d+h+N-1+k_{2}+A\right) \pi-\frac{\tilde{k}_{3}}{\sqrt{s}} .
\end{aligned}
$$

Thus from Corollary 2.8 we have

$$
\begin{aligned}
& \left(m+\tilde{k}_{2}-1\right) \pi+\tan ^{-1} \nu_{2 k+3}-\frac{\tilde{L}_{2 k+2}\left(\tilde{k}_{3}\right)}{\sqrt{s}} \\
& \quad<\Omega_{2 k+4}(s) \\
& \quad<\left(m+N+\tilde{k}_{2}\right) \pi+\tan ^{-1} \nu_{2 k+3}+\frac{\tilde{L}_{2 k+2}\left(\tilde{k}_{3}\right)}{\sqrt{s}},
\end{aligned}
$$

which completes the proof of the lemma.

The following lemma can be proved in a manner similar to Lemma 4.1 its proof is omitted.

Lemma 4.2. Let $r<-c<0$ on $\left(a_{2 j}, a_{2 j+1}\right), j=0, \ldots, k$, and $r>c>0$ on $\left(a_{2 j-1}, a_{2 j}\right), j=1, \ldots, k$. Let $s_{0} \leq s_{1} \leq s_{2} \leq \ldots$ be a listing of

$$
\Sigma=\bigcup_{i=1, \ldots, 2 k+1}^{\bullet} \sigma_{i}
$$

where each element is repeated according to its multiplicity. There exist constants $k_{1}, k_{2} \in \mathbb{Z}$ and $k_{0}, k_{3}>0$ such that if $m \geq k_{1}$, then

$$
s \in P_{m} \quad \Longrightarrow \quad \Omega_{2 k+1} \in \tilde{J}_{m+k_{2}}\left(2 k-1, s, k_{3}\right),
$$

where $P_{m}=\left[s_{m}+\frac{k_{0}}{\sqrt{s_{m}}}, s_{m+1}-\frac{k_{0}}{\sqrt{s_{m+1}}}\right]$, and, for any $m$ such that $P_{m-1} \neq \emptyset$ and integers $N \geq 0$ such that $P_{m+N} \neq \emptyset$,

$$
s \in\left(s_{m}-\frac{k_{0}}{\sqrt{s_{m}}}, s_{m+N}+\frac{k_{0}}{\sqrt{s_{m+N}}}\right) \Longrightarrow \Omega_{2 k+1} \in \bigcup_{i=m}^{m+N} \tilde{J}_{i-1+k_{2}}^{\prime}\left(2 k-1, s, k_{3}\right),
$$

where $\Omega_{2 k+1}=\Omega\left(a_{2 k+1}, s, 0, \Omega_{0}\right)$ is the modified Prüfer angle at $a_{2 k+1}$, given that the modified Prüfer angle at $a_{0}$ is given by (3.1).

\section{Proof of Theorem 1.2}

We are now ready to prove our main result.

Note 5.1. For $s>0, \lambda=s^{2}$ is an eigenvalue of (1.1), (1.2), (1.3) if and only if:

(a) for the case of $r<0$ on $\left(a_{n-1}, a_{n}\right)$,

$$
\Omega_{n}=\Omega\left(a_{n}, s, 0, \Omega_{0}\right)=\Phi_{n}+m \pi, \quad m \in \mathbb{Z},
$$

where $\Omega_{0}$ and $\Phi_{n}$ satisfy (3.1) and (3.2) respectively;

(b) for the case of $r>0$ on $\left(a_{n-1}, a_{n}\right)$,

$$
\Omega_{n-1}=\Omega\left(a_{n-1}, s, 0, \Omega_{0}\right)=\Phi_{n-1}+m \pi, \quad m \in \mathbb{Z},
$$


where $\Phi_{n-1}$ is as in Lemma 3.2 .

Moreover (cf. Lemma 3.4), the oscillation count of non-trivial solutions of (1.1), with boundary condition given by (1.2) and (1.3) when $m$ is as given by (a) or (b), is

$$
\begin{array}{r}
m+1 \quad \text { if } \quad \alpha<0 \quad \text { and } \quad \beta>0, \\
m \quad \text { if } \quad \alpha \geq 0 \quad \text { and } \quad \beta>0, \\
m \quad \text { if } \quad \alpha<0 \quad \text { and } \quad \beta \leq 0, \\
m-1 \quad \text { if } \quad \alpha \geq 0 \quad \text { and } \quad \beta \leq 0 .
\end{array}
$$

Proof of Theorem 1.2. As before we shall restrict attention to the case of $\alpha, \beta \in$ $(0, \pi / 2]$, the other cases being similar. if

We begin with the case of $r<0$ on $\left(a_{n-1}, a_{n}\right)$. From Note 5.1 $s \in \sigma$ if and only

$$
\Omega_{n}=\Phi_{n}+m \pi, \quad m \in \mathbb{Z},
$$

where $m$ is the oscillation count associated with $s$.

For sufficiently large $m$, there is a unique solution $s$ to (5.1) for each large $m \in \mathbb{N}$, by Sturm's Theorem. This solution corresponds to the intersection of the graphs of $\Phi_{n}$ and $\Omega_{n}-m \pi$. From Lemmas 4.1 and 4.2 (corresponding to even and odd $n$ respectively) we see that $\Omega_{n}-m \pi$ and $\Phi_{n}$ are as illustrated in Figure 4 for large $s$. Here the $s_{m}$ form a listing of the disjoint union of $\sigma_{0}, \ldots, \sigma_{n-1}$ with repetition according to multiplicity. Note that from (3.4) we obtain $\Phi_{n}=\frac{\pi}{2}+O(1 / s)$, and from Corollary 2.8 the $\tilde{J}_{k}$ are bounded away from $\pi / 2$ for large $s$. This case of the theorem has thus been established.

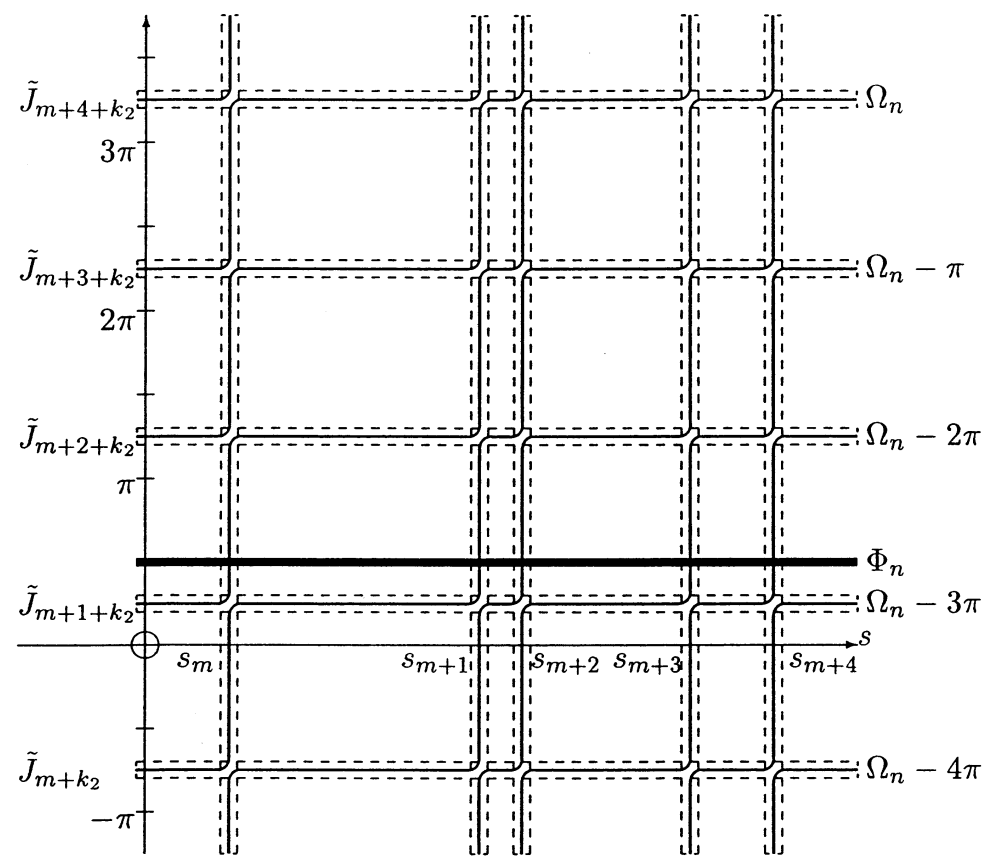

FIgURE $4 . \Phi_{n}$ and $\Omega_{n}-m \pi$ as functions of $s$ 

if

We proceed to the case of $r>0$ on $\left(a_{n-1}, a_{n}\right)$. From Note 5.1 $s \in \sigma$ if and only

$$
\Omega_{n-1}=\Phi_{n-1}+m \pi, \quad m \in \mathbb{Z}
$$

and again $m$ is the oscillation count corresponding to $s$. From Lemma3.3(b), there are positive constants $c^{-}$and $c^{+}$such that for large $s$

$$
c^{-} \leq-\dot{\Phi}_{n-1} \leq c^{+},
$$

where ${ }^{\cdot}=\frac{\partial}{\partial s}$. Denote by $s_{0} \leq s_{1} \leq \ldots$ a listing in increasing order, with repetition according to multiplicity, of the disjoint union of $\sigma_{0}, \ldots, \sigma_{n-3}$ (note that $\sigma_{n-2}=\emptyset$ ). Then with Lemmas 4.1 and 4.2 (corresponding to odd and even $n$ respectively) we have, for large $s$, the situation illustrated in Figure 5 . As before, there is a unique intersection between the graphs of $\Omega_{n-1}-m \pi$ and $\Phi_{n-1}$, by Sturm's Theorem.

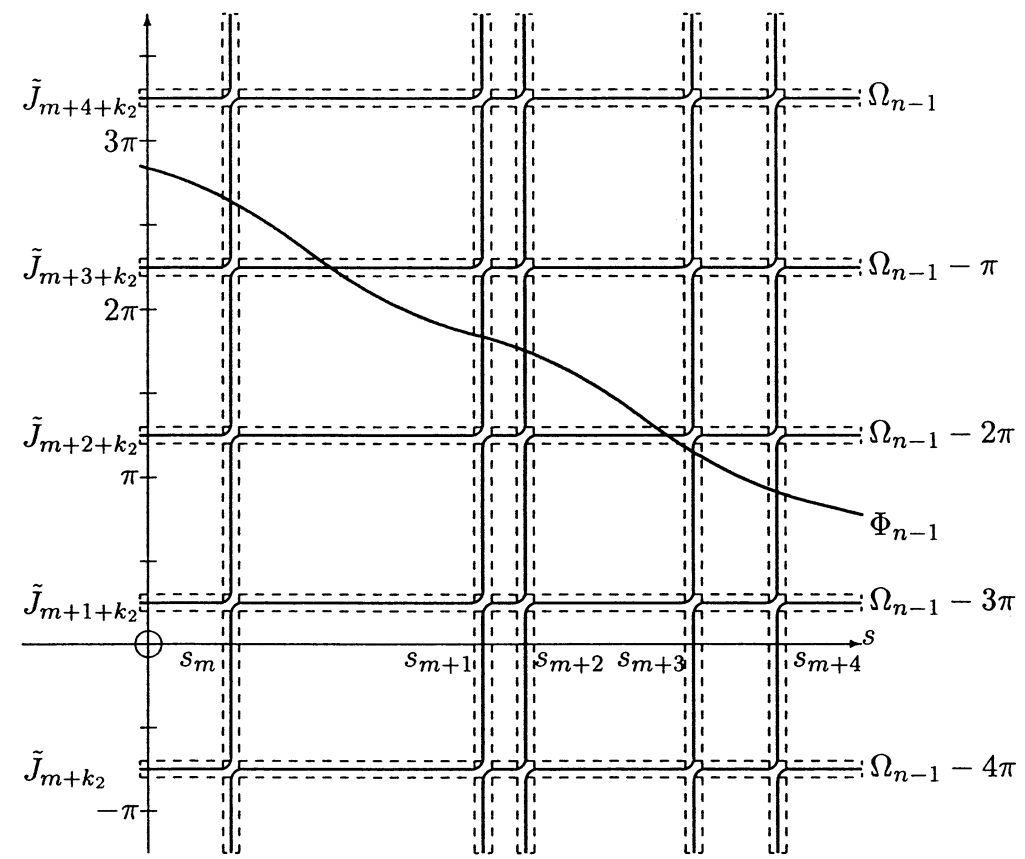

FIGURE 5. $\Phi_{n-1}$ and $\Omega_{n-1}-m \pi$ as functions of $s$

It is apparent from Figure 5 that the ordered sequence $\sigma$ can be partitioned, not necessarily uniquely, into two subsequences $t_{i}$ and $\eta_{j}$, in such a manner that

$$
t_{i} \in\left(s_{i}-\frac{k_{0}}{\sqrt{s_{i}}}, s_{i}+\frac{k_{0}}{\sqrt{s_{i}}}\right)
$$

and

$$
\Phi_{n-1}\left(\eta_{j}\right) \in \tilde{J}_{j}\left(n-3 ; \eta_{j} ; k_{3}\right),
$$

where $k_{0}$ and $k_{3}$ are as given in Lemmas 4.1 and 4.2. Thus it suffices to show that the $\eta_{j}$ are within $O\left(\frac{1}{\sqrt{3}}\right)$ of the eigenvalues in $\sigma_{n-1}$.

From (5.2), (5.4) and Corollary 2.8.

$$
\eta_{j}=\Phi_{n-1}^{-1}\left(j \pi+\tan ^{-1} \nu_{n-2}+O\left(1 / \sqrt{\eta_{j}}\right)\right)=\mu_{j}+O(1 / \sqrt{j}),
$$


cf. [1], where $\mu_{j}=\Phi_{n-1}^{-1}\left(j \pi+\tan ^{-1} \nu_{n-2}\right) \in \sigma_{n-1}$, since (1.9) and (1.3) are equivalent to $\tan \Phi_{n-1}=\nu_{n-2}$ for $j=n-1$. This completes the proof.

\section{EXAMPLE}

We present an example illustrating our theory. On $[-3,3]$ we set $q(x)=0$ and

$$
r(x)= \begin{cases}1, & x \in[-3,-2] \cup[-1,1] \cup[2,3], \\ -1, & x \in(-2,-1) \cup(1,2),\end{cases}
$$

and consider the eigenvalue problem

$$
\begin{aligned}
-y^{\prime \prime} & =s^{2} r y, \\
y^{\prime}(-3)=0 & =y^{\prime}(3) .
\end{aligned}
$$

We begin by solving the problem directly. For $x \in[-3,-2]$, the differential equation is $-y^{\prime \prime}=s^{2} y$, which, subject to the Neumann condition at $x=-3$, has solution $y(x)=\cos s(x+3)$. Thus

$$
\left[\begin{array}{c}
y \\
y^{\prime}
\end{array}\right](-2)=\left[\begin{array}{c}
\cos s \\
-s \sin s
\end{array}\right]
$$

Across $[-2,-1]$ the solution now takes the form

$$
y=\cos s \cosh s(x+2)-\sin s \sinh s(x+2),
$$

leading to

$$
\left[\begin{array}{c}
y \\
y^{\prime}
\end{array}\right](-1)=\left[\begin{array}{c}
A(s) \\
s B(s)
\end{array}\right]
$$

where

$$
\begin{aligned}
& A(s)=\cos s \cosh s-\sin s \sinh s \\
& B(s)=\cos s \sinh s-\sin s \cosh s .
\end{aligned}
$$

Continuing to $[-1,1]$, we find the solution to be

$$
y(x)=A(s) \cos s(x+1)+B(s) \sin s(x+1) .
$$

The symmetry of the problem can be invoked to frame the eigencondition for the solution constructed thus far as $y^{\prime}(0) y(0)=0$. Considering $y(0)=0$, we obtain

$$
A(s) \cos s+B(s) \sin s=0 .
$$

It is easy to check that $\cos s \neq 0$ for (6.3) to hold, and so

Hence

$$
\tan s=-\frac{A(s)}{B(s)}=\frac{-1+\tan s \tanh s}{\tanh s-\tan s} .
$$

$$
\begin{aligned}
\tan s \tanh s-\tan ^{2} s & =-1+\tan s \tanh s, \\
\tan s & = \pm 1,
\end{aligned}
$$

showing that $s=\left(n+\frac{1}{4}\right) \pi,\left(n+\frac{3}{4}\right) \pi, n \geq 0$.

The condition $y^{\prime}(0) \stackrel{=}{=}$ yields $s=0$, or

$$
\begin{gathered}
\tan s=\frac{B(s)}{A(s)}=\frac{\tanh s-\tan s}{1-\tan s \tanh s} \\
\tanh s \tan ^{2} s-2 \tan s+\tanh s=0, \\
\tan s=\frac{1 \pm \sqrt{1-\tanh ^{2} s}}{\tanh s} .
\end{gathered}
$$


Now for large $s$ we have tanh $s=1-\eta$, where $\eta=O\left(e^{-2 s}\right), \eta>0$; so (6.4) gives

$$
\begin{aligned}
\tan s & =1 \pm \epsilon, \quad \epsilon>0, \quad \epsilon=O\left(e^{-s}\right), \\
s & =\left(n+\frac{1}{4}\right) \pi \pm \epsilon_{n}, \quad \epsilon_{n}>0, \quad \epsilon_{n}=O\left(e^{-n}\right), n \geq 0 .
\end{aligned}
$$

The upshot of these calculations is that the spectrum of the problem (6.1)-(6.2) is given by

$$
\sigma=\{0\} \cup\left\{\left(n+\frac{1}{4}\right) \pi \pm \epsilon_{n},\left(n+\frac{1}{4}\right) \pi,\left(n+\frac{3}{4}\right) \pi: n \geq 0, \epsilon_{n}>0, \epsilon_{n}=O\left(e^{-n}\right)\right\} .
$$

In terms of our general theory we have $a_{0}=-3, a_{1}=-2, a_{2}=-1, a_{3}=1$, $a_{4}=2, a_{5}=3, \nu_{j}=1=\epsilon_{j}, 0 \leq j \leq 4, \zeta_{j}=1, j=0,1,3,4$, and $\zeta_{2}=2$.

To compute $\sigma_{0}$ we solve the problem

$$
\begin{array}{r}
-y^{\prime \prime}=s^{2} y \quad \text { on }(-3,-2), \\
y^{\prime}(-3)=0, \quad s y(-2)=-y^{\prime}(-2) .
\end{array}
$$

It is straightforward to check that this requires $s=0$ or $\tan s=1$, i.e., $s=0$ or $\left(n+\frac{1}{4}\right) \pi, n \geq 0$.

For $\sigma_{2}$ we have the problem

$$
\begin{array}{r}
-y^{\prime \prime}=s^{2} y \quad \text { on }(-1,1), \\
s y(-1)=y^{\prime}(-1), \quad s y(1)=-y^{\prime}(1) .
\end{array}
$$

Again, easy calculations give $s=0$ or $\left(n+\frac{1}{4}\right) \pi,\left(n+\frac{3}{4}\right) \pi, n \geq 0$.

Finally, for $\sigma_{4}$ we have

$$
\begin{aligned}
-y^{\prime \prime}=s^{2} y & \text { on }(2,3), \\
s y(2)=y^{\prime}(2), & y^{\prime}(1)=0,
\end{aligned}
$$

which produces $s=0,\left(n+\frac{1}{4}\right) \pi, n \geq 0$.

It is readily seen that $\sigma_{0}, \sigma_{2}, \sigma_{4}$ satisfy the conclusions of Corollary 3.6, and that $\sigma$ of (6.5) is the disjoint union

$$
\sigma_{0} \dot{\cup} \sigma_{2} \dot{\cup} \sigma_{4}=\left\{0,0,0, \frac{\pi}{4}, \frac{\pi}{4}, \frac{\pi}{4}, \frac{3 \pi}{4}, \frac{5 \pi}{4}, \frac{5 \pi}{4}, \frac{5 \pi}{4}, \frac{7 \pi}{4}, \frac{9 \pi}{4}, \frac{9 \pi}{4}, \ldots\right\}
$$

to exponentially small order (recall $\sigma_{1}=\sigma_{3}=\emptyset$ ), illustrating Theorem 1.2 in a case of resonance.

\section{ACKNOWLEDGEMENTS}

The research of P. A. Binding and P. J. Browne was supported in part by grants from the NSERC of Canada. The research of B. A. Watson was conducted while visiting the Universities of Calgary and Saskatchewan, and was supported in part by the Centre for Applicable Analysis and Number Theory.

\section{REFERENCES}

[1] F. V. Atkinson, A. B. Mingarelli, Asymptotics of the number of zeros and the eigenvalues of general weighted Sturm-Liouville problems, J. reine angewandte Math. (1987) 375/376, 380-393. MR 88d:34023

[2] P. A. Binding, P. J. Browne, K. Seddighi, Sturm-Liouville problems with eigenparameter dependent boundary conditions, Proc. Edinburgh Math. Soc. (1993) 37, 57-72. MR 95k:34039

[3] E. A. Coddington, N. Levinson, Theory of Ordinary Differential Equations, McGraw-Hill, 1955. MR 16:1022b 
[4] A. A. Dorodnicyn, Asymptotic laws of distribution of the characteristic values for certain special forms of differential equations of the second order, Amer. Math. Soc. Translations, Series 2, (1960) 16, 1-101. MR 22:8161

[5] W. Eberhard, G. Freiling, The distribution of the eigenvalues for second order eigenvalue problems in the presence of an arbitrary number of turning points, Results in Math. (1992) 21, 24-41. MR 93e:34108

[6] G. M. Guabreev, V. N. Pivovarchik, Spectral analysis of the Regge problem with parameters, Funct. Anal. Appl. (1997) 31, 54-57. MR 98g:34134

[7] H. Hochstadt, On inverse problems associated with Sturm-Liouville operators, J. Differential Equations (1975) 17, 220-235. MR 51:3601

[8] E. L. Ince, Ordinary Differential Equations, Dover, 1944. MR 6:65f

[9] R. E. Langer, On the asymptotic solutions of ordinary differential equations, with an application to the Bessel functions of large order, Trans. Amer. Math. Soc. (1931) 33, 23-64

[10] B. M. Levitan, M. G. Gasymov, Determination of a differential equation by two of its spectra, Russian Math. Surveys (1964) 19, no. 2, 1-64. MR 29:299

[11] S. Strelitz, Asymptotics for solutions of linear differential equations having turning points with applications, Mem. Amer. Math. Soc. (1999) 142, Number 676. MR 2000e:34086

Department of Mathematics and Statistics, University of Calgary, Calgary, AlBERTA, CANADA T2N 1N4

E-mail address: binding@ucalgary.ca

Mathematical Sciences Group, Department of Computer Science, University of Saskatchewan, Saskatoon, Saskatchewan, Canada S7N 5E6

E-mail address: browne@snoopy.usask.ca

Department of Mathematics, University of the Witwatersrand, Private Bag 3, P O WITS 2050, South AfricA

E-mail address: watson-ba@e-math.ams.org 